Case Report

\title{
Acute Hemorrhagic Myositis in Inflammatory Myopathy and Review of the Literature
}

\author{
Howard Van Gelder, ${ }^{1}$ Kim M. Wu, ${ }^{1}$ Nayiri Gharibian, ${ }^{2}$ Dharmi B. Patel, ${ }^{2}$ \\ Philip J. Clements, ${ }^{1}$ Emil R. Heinze, ${ }^{1,2}$ Robert I. Morris, ${ }^{1}$ and Andrew L. Wong ${ }^{1,2}$ \\ ${ }^{1}$ UCLA-Olive View Rheumatology Program, Division of Rheumatology, Olive View-UCLA Medical Center, \\ 14445 Olive View Drive, 2B182, Sylmar, CA 91342, USA \\ ${ }^{2}$ UCLA-Olive View Internal Medicine Program, Department of Medicine, Olive View-UCLA Medical Center, \\ 14445 Olive View Drive, 2B182, Sylmar, CA 91342, USA
}

Correspondence should be addressed to Andrew L. Wong; alunwong@ucla.edu

Received 15 July 2014; Revised 22 September 2014; Accepted 22 September 2014; Published 14 October 2014

Academic Editor: Masataka Kuwana

Copyright (C) 2014 Howard Van Gelder et al. This is an open access article distributed under the Creative Commons Attribution License, which permits unrestricted use, distribution, and reproduction in any medium, provided the original work is properly cited.

We describe two patients with dermatomyositis that presented with interstitial lung disease, positive V and Shawl sign who developed acute spontaneous abdominal/retroperitoneal bleed. Both patients expired despite aggressive treatment and resuscitation. Hemorrhagic myositis in these two patients with inflammatory myopathy is a very rare complication. The association of anti-Ro52 with this potentially very serious complication remains unclear. This potential relationship should be further evaluated in future studies.

\section{Introduction}

Dermatomyositis is an autoimmune idiopathic inflammatory myopathy (IIM) with characteristic skin manifestations, classically heliotrope rash, V, shawl, and Holster sign, and Gottron's papules [1]. Up to 30 percent of patients with dermatomyositis (DM) or polymyositis (PM) have a constellation of clinical findings termed the "antisynthetase syndrome" [2-4]. Findings include constitutional symptoms such as fever, interstitial lung disease (ILD), myositis, Raynaud's phenomenon, mechanic's hands, and arthritis [5]. Dermatomyositis can be associated with systemic manifestations including ILD, pneumomediastinum, and cardiomyopathy [6]. We present two cases of inflammatory myopathy (Dermatomyositis) complicated by acute spontaneous hemorrhage of the iliopsoas, psoas, and pectineus muscles. To our knowledge, there are only a handful of cases of myositis complicated by hemorrhage.

\section{Case 1}

A 50-year-old African-American male with a history of borderline diabetes presented with three weeks of myalgia, a fifteen-pound weight loss, arthralgia, odynophagia, fatigue, dyspnea on exertion, and progressive nonproductive cough in early 2012. These symptoms coincided with a new erythematous rash on the back of his scalp, neck, and inner left ear and over the knuckles of his hands. A short trial of $4 \mathrm{mg}$ of oral methylprednisolone was given at a previous urgent care visit with some improvement in the rash. Review of systems confirmed intermittent subjective fever, hoarseness, and tea color urine for 4 days. Medications included over-thecounter smooth move for constipation, multivitamins, and fish oil.

Initial exam showed normal vital signs. His voice was hoarse. His lung exam revealed mild crackles bilaterally. Muscle strength testing of the neck flexors and proximal upper and lower extremities was $4 / 5$ with bilateral distal upper and lower extremities scoring 5/5. Skin exam showed malar erythema, erythematous patches over the scalp and behind the ears, minimal heliotrope rash, mild erythema over his chest (V sign), and diffuse erythematous hypopigmented macules over his proximal interphalangeal joints (PIPs) and metacarpal phalangeal joints (MCPs) bilaterally. $\mathrm{He}$ had "mechanic's hands" and periungal erythema. There was 


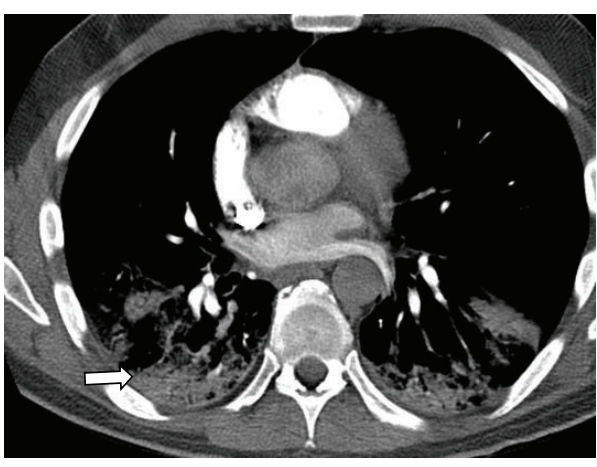

(a)

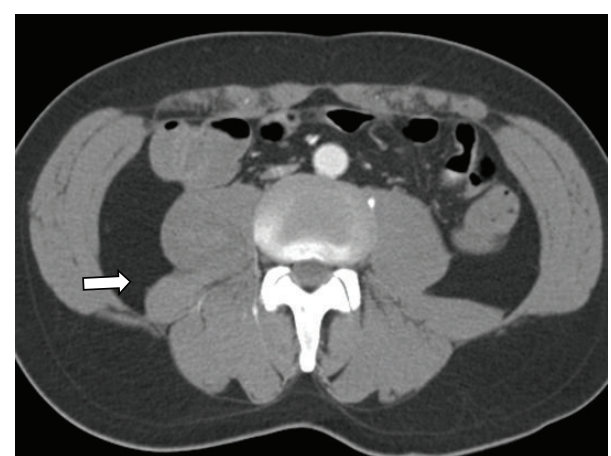

(b)

FIgURE 1: (a) CT chest on admission. Axial contrast enhanced images through the chest in the pulmonary phase demonstrate a normal main pulmonary artery trunk and normal segmental pulmonary arterial branches extending to the superior segment of the right upper lobe. Lung windows reveal lower lobe consolidation (see arrow). (b) Normal appearance of the retroperitoneum at that time (see arrow).

a small hard papule along the medial aspect of the right 3rd PIP, which was suspected of being calcinosis.

Initial laboratory results revealed elevated creatine phosphokinase (CPK) of 1123 units/L (reference: 26-174 units/L) with mild elevation of transaminases but unremarkable complete blood count (CBC), chemistry panel, and coagulation studies. Chest X-ray showed patchy consolidations of the right upper lung and posterior lung bases suggestive of possible pneumonia. CT chest showed right upper lobe groundglass opacity and bilateral lower lobe consolidations with mildly enlarged mediastinal lymph nodes (Figure 1). He was started on ceftriaxone and azithromycin for empiric coverage of community acquired pneumonia. He was admitted for treatment and workup of a possible inflammatory myopathy.

During the initial hospitalization, the patient's CPK improved with bed rest and normal saline infusion. CT abdomen and pelvis ordered to evaluate persistent constipation was unremarkable except for a small hepatic cyst. EMG/NCS showed no electrodiagnostic evidence of a polyneuropathy or myopathy. MRI-STIR of the thighs showed no convincing evidence of an inflammatory myopathy other than minimal muscle edema of the iliopsoas muscles. Given no clear focus of inflammatory muscle seen on MRI or EMG, a muscle biopsy was deferred. For continued hoarseness of voice, laryngoscopy was performed and showed postcommissure edema and erythema involving the laryngeal epiglottis and false cords. For evaluation of continuing dyspnea with gradual worsening hypoxia, a bronchoscopy was performed that visualized signs of mild inflammation in the lungs. Bronchoalveolar lavage returned numerous WBCs with normal respiratory flora present. Transbronchial biopsy of the right lower lung was consistent with organizing pneumonia and was negative for fungi and acid fast bacilli (Figure 2). A transthoracic echocardiogram was read as within normal limits and a CT angiogram was negative for pulmonary embolism. Immunofluorescence for antinuclear antibodies (ANA), serum for anti-RNP/Sm, antiphospholipid antibodies, anti-Mi-2, anti-SRP, anti-Jo-1, antineutrophil cytoplasmic antibodies (ANCA), HIV, hepatitis panel, aldolase, and sputum for $\mathrm{AFBx} 3$ all returned normal.

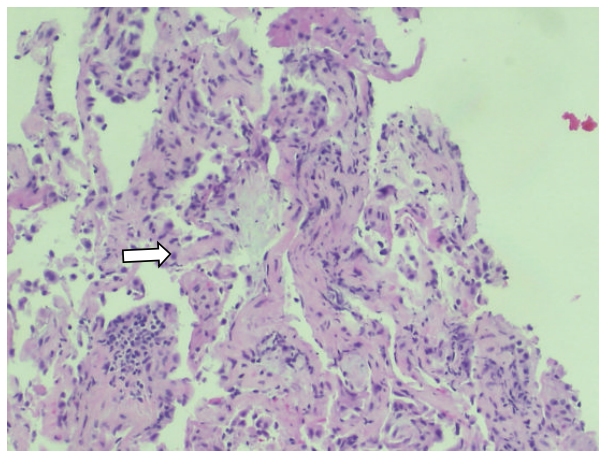

FIGURE 2: Lung biopsy (mild chronic interstitial pneumonitis with focal fibroblastic foci, consistent with organizing pneumonia; stains for fungi and AFB were negative) (see arrow).

In the interim, the patient developed persistent fever thought likely to be secondary to an underlying inflammatory process. Solumedrol $60 \mathrm{mg}$ IV q 12 hours was started after one week into his hospitalization with near immediate resolution of fevers and improvement of the rash. Given the likely diagnosis of dermatomyositis, two doses of intravenous immunoglobulin (IVIG) were given to treat his persistent dysphagia and unresolving organizing pneumonia. Patient reported improved strength after receiving IVIG and steroid therapy, but he remained hypoxic. Repeat CT scans of chest/abdomen/pelvis were performed and showed no progression of disease on day 15 (Figure 3). On day 17 of hospitalization, the patient began complaining of right hip pain for which an MRI was ordered to rule out avascular necrosis. Later that evening, the patient was found to be uncomfortable in bed and only partially responsive. He became hypotensive, tachycardic, and tachypneic. CBC revealed hemoglobin of $4.1 \mathrm{gm} / \mathrm{dL}$ and platelet count of $50,000 / \mu \mathrm{L}$. The patient was intubated, started on vasopressors, and transferred to the intensive care unit (ICU). He was aggressively resuscitated with IV fluid and blood products. He was also noted to have increasing abdominal girth. Stat CT abdomen/pelvis showed 


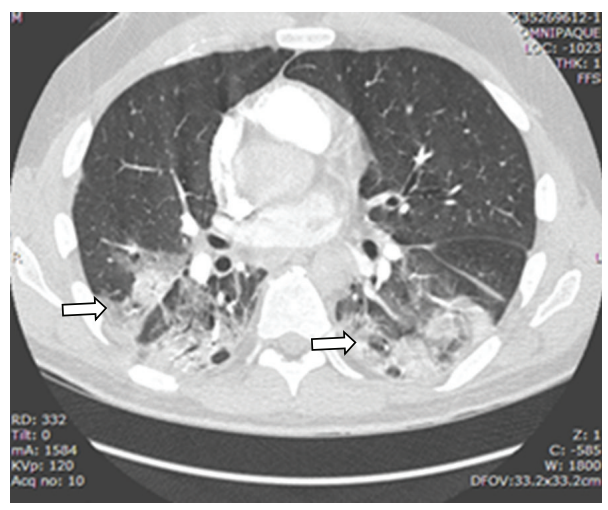

(a)

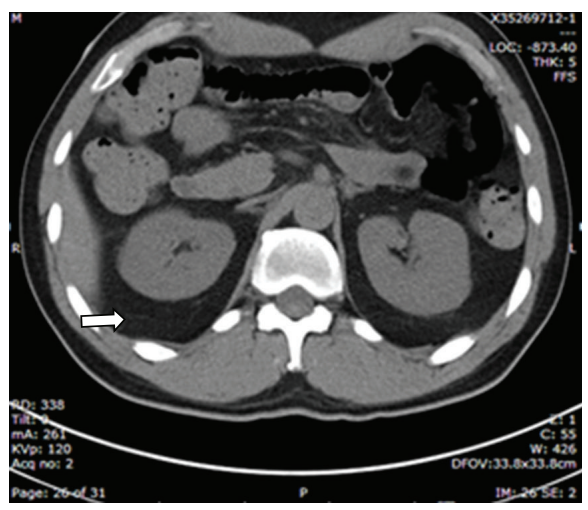

(b)

FIGURE 3: (a) CT chest/abdomen/pelvis. No evidence for pulmonary embolism. Patchy peribronchiolar consolidations are worsened in the right upper lobe, right lower lobe, and left lower lobe (see arrow). (b) No abdominal masses/hemorrhage is seen (see arrow).

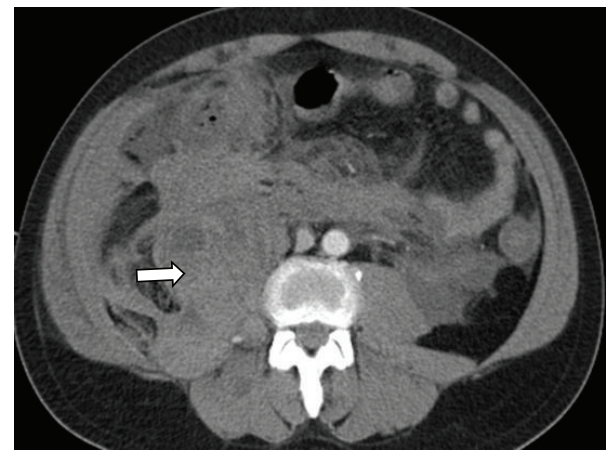

FIGURE 4: Stat CT abdomen/pelvis. Interval development of a large retroperitoneal hemorrhage centered around the right psoas muscle, with extension to the right spinous erector and iliacus musculature (see arrow).

large retroperitoneal bleeding involving the right iliacus and psoas muscles, extending into the retroperitoneum and along the right paracolic gutter (Figure 4). Interventional radiology could not locate a focal source of bleeding and suggested aggressive medical resuscitation. Solumedrol was also increased to $250 \mathrm{mg}$ IV Q6 for possible dermatomyositis associated vasculitis. The patient received a total of 20 units of pack red blood cells, fresh frozen plasma, cryoprecipitate, and 3 units of platelets but ultimately progressed into multiorgan failure. On day 19, the patient was found to be in asystole and expired that day.

The family subsequently agreed to a partial autopsy limited to the chest and abdomen. The autopsy results showed a massive retroperitoneal hematoma, segmental myofiber necrosis in the psoas muscle without lymphocytic infiltrate, and perifascicular atrophy or necrosis (Figure 5). The patient's serum was sent to a reference lab where anti-Ro52 returned positive.

\section{Case 2}

A 63-year-old Filipino female with a history of diabetes, hypertension, and hyperlipidemia presented to our hospital for new throat pain, fevers, and oral sores for four weeks in early 2013. She had difficulty swallowing to the point where she could no longer eat solid foods and had lost 10-15 lbs. She developed new painful mouth sores involving her lips along with low grade subjective fevers and joint swelling of the fingers during the same time period. She reported a new rash around her eyes and chest that was not itchy or painful. She complained of worsening fatigue over the past week, required assistance to eat and go to the bathroom, and was bedbound on presentation. ROS confirmed a dry cough for four weeks not improved with oral azithromycin prescribed by her PMD. She had been seen in the ED one month ago for the joint pains where a hand XR reportedly showed osteoarthritis of her hands and a chest X-ray noted prominent interstitial lung markings. Medications included amlodipine, metformin, quinapril, ezetimibe, glipizide, aspirin, calcium, and fish oil.

Upon presentation she was afebrile with a blood pressure of $140 / 80 \mathrm{mmHg}$, pulse of 107 beats/minute, respiratory rate of 26 breaths/minute, and oxygen saturation of $86 \%$ on room air that improved to $95 \%$ with $2 \mathrm{~L}$ nasal cannula oxygen. She was found to be severely hyponatremic with poor PO intake and have a new bilateral infiltrate on CT angiogram (Figure 6) of the chest, and thus she was admitted for further care. Initial exam was significant for hoarse voice, oropharyngeal ulcerations/erythema, heliotrope rash around the eyes, positive shawl and $\mathrm{V}$ sign, and bilateral crackles at the lung bases. Her hand examination showed distal cracking of her skin (mechanic's hands) along with purpuric lesions. Strength testing showed initially $5 / 5$ in all muscle groups but decreased in the lower extremities to $3 / 5$ early in the course of her hospital stay.

Initial labs were significant for CPK level of $2246 \mathrm{IU} / \mathrm{I}$, aldolase of $14.2 \mathrm{IU} / \mathrm{L}, \mathrm{LDH} 498 \mathrm{IU} / \mathrm{L}, \mathrm{AST} 310 \mathrm{IU} / \mathrm{L}$, ALT $77 \mathrm{IU} / \mathrm{L}$, alkaline phosphatase $309 \mathrm{IU} / \mathrm{L}, \mathrm{T}$. bilirubin $0.7 \mathrm{mg} / \mathrm{dL}$, and CRP $41.5 \mathrm{mg} / \mathrm{mL}$. All testing for bacterial/ fungal pneumonia, urine histoplasmosis antigen, viral hepatitis antibody/antigens, aspergillosis antigen, legionella antigen, influenza A/B antibody, syphilis (RPR) antibody, coccidioidomycosis antibody, and cryptococcus antibody 


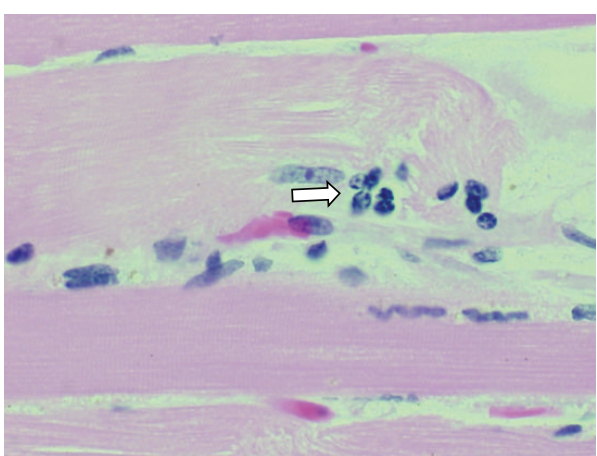

(a)

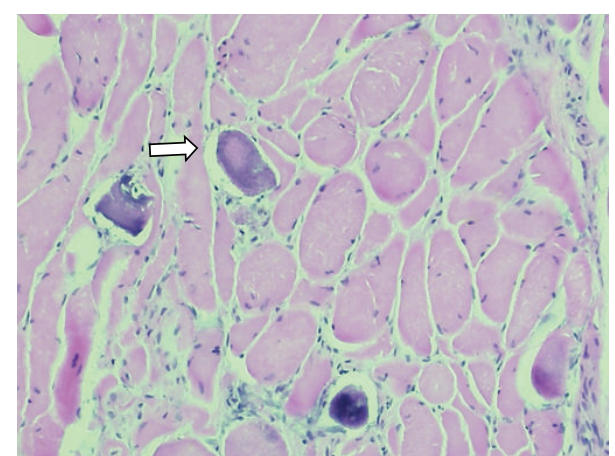

(b)

Figure 5: (a) Autopsy of the psoas muscle. Skeletal muscles: nonuniform degree of interior scattered segments of necrotic myofibers in the psoas fascicles without perifascicular myofiber atrophy or necrosis. Extensive morular aggregation of myofiber nuclei in the damaged myofibers (see arrow). Minimal perimysial and endomysial fibrosis, suggesting an acute process. Finding may suggest possible sepsis with intravascular coagulation and capillary disruption. (b) Calcium deposition found in myofiber (see arrow).

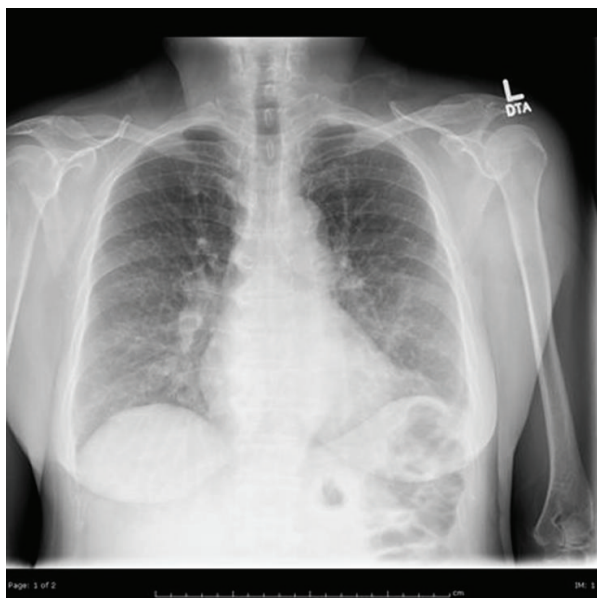

(a)

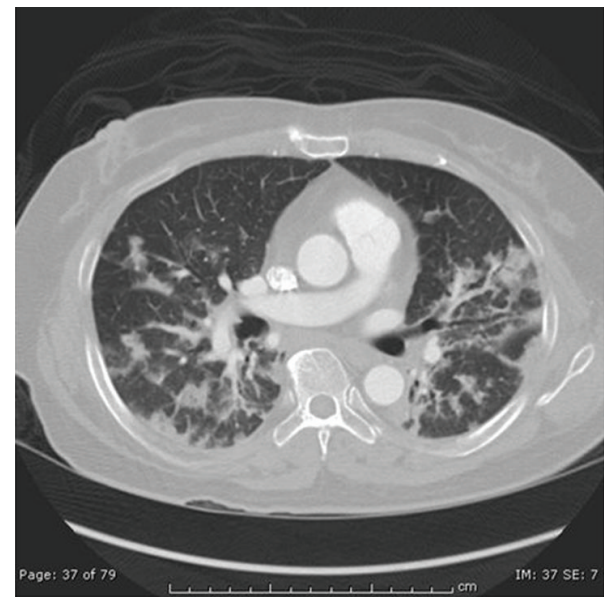

(b)

FIGURE 6: Lung imaging on admission. (a) Chest radiograph showing prominent interstitial lung markings. (b) CT pulmonary angiogram showing multifocal opacities and consolidation.

was negative. ANA, anti-RNP/Sm, anti-dsDNA, anti-Scl70, anti-centromere $\mathrm{B}$, anti-smooth muscle, anti-histone, antiCCP, ANCA, anti-Mi-2, anti-Jo-1, and anti-SSB antibodies were all negative. Anti-SSA was mildly positive at $>1.2$ (normal < 1.0 IU). Magnetic resonance imaging (MRI) with short tau inversion recovery (STIR) imaging of the pelvis and shoulders showed diffuse edema in symmetric distribution throughout the shoulder girdles and pelvis bilaterally (Figure 7). Electromyogram with nerve conduction study revealed sharp waves and irritable myopathy consistent with inflammatory myopathy.

A diagnosis of dermatomyositis was made based on the presence of typical rash, mechanic's hands, muscle weakness, elevated muscle enzymes, abnormal EMG/MRI studies, and probable interstitial lung disease. The patient was started on solumedrol $80 \mathrm{mg}$ IV BID and empiric levofloxacin for possible pneumonia. Her CPKs downtrended and her facial and body rashes began to resolve but she remained weak with worsening hypoxia and dysphonia. Given the aggressive nature of her disease, she was given IVIG infusion on consecutive days beginning approximately six days after admission while awaiting a planned muscle biopsy. She had a CT abdomen and pelvis, to search for possible malignancy during the second day of her IVIG infusion, which was read as negative for acute pathology (Figures $8(a)-8(c)$ ). One day after finishing her second dose of IVIG, she became altered with a rapid drop of $\mathrm{Hgb}$ from 10.3 to $6.1 \mathrm{gm} / \mathrm{dL}$. A repeat CT scan of the abdomen and pelvis revealed new areas of intramuscular hematomas that were not seen 24 hours ago on the prior CT (Figures $8(\mathrm{~d})-8(\mathrm{f})$ ). During her hospitalization, she had only been on prophylactic doses of subcutaneous dalteparin of 5000 units/day with a normal platelet count of 176 on the day of the bleeding event with an INR of 1.21 seconds on admission. Steroid therapy was increased to full 1-gram pulse doses and one dose of IV Cytoxan was given, but eventually the patient slowly decompensated and died of 


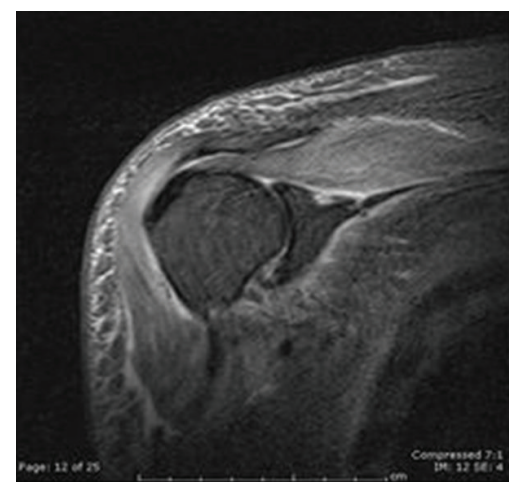

(a)

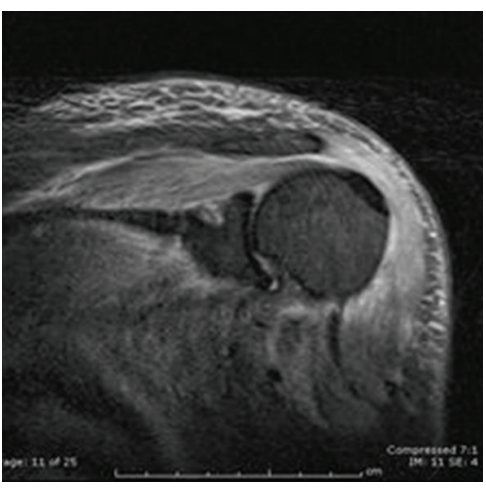

(b)

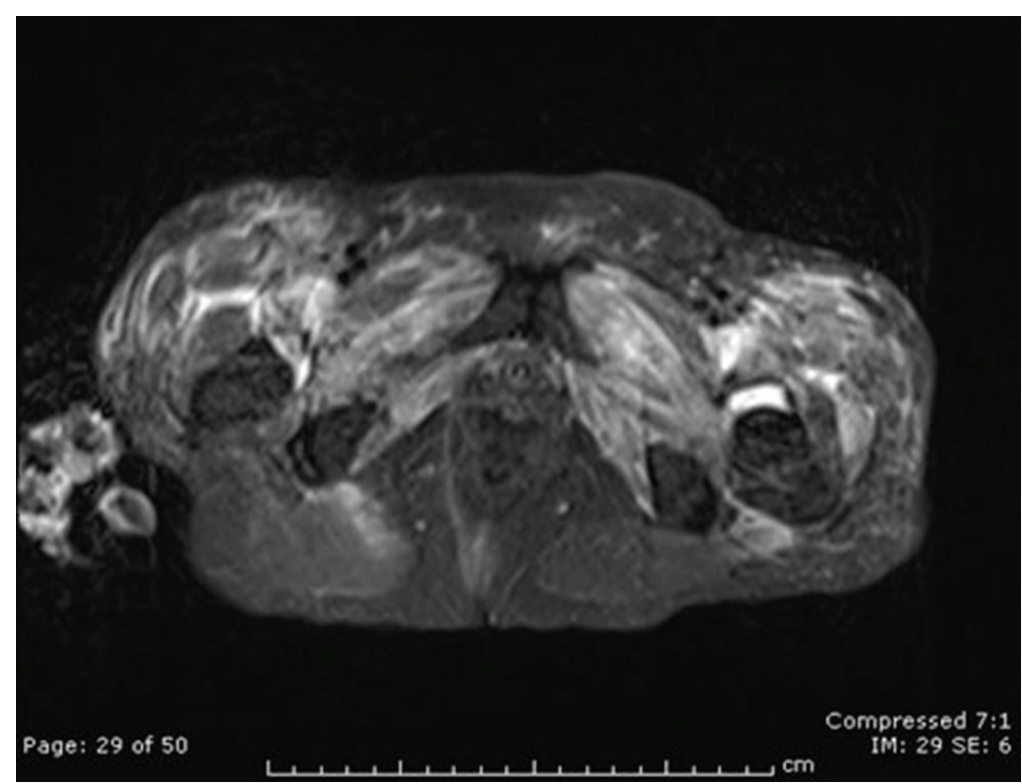

(c)

FIGURE 7: MRI with short tau inversion recovery (STIR) image showing diffuse symmetrical proximal muscle edema. (a) Right shoulder, (b) left shoulder, and (c) pelvis.

multiorgan failure a week later. A sample of her serum early in her hospital course was sent to a reference lab where notably she tested positive for anti-Ro/SSA-52 antibodies.

\section{Discussion}

Here we present two cases of idiopathic inflammatory myopathy (dermatomyositis) complicated by acute severe hemorrhagic myositis. The diagnosis of hemorrhagic myositis is rare and has been reported only several times in the literature but is a serious, often fatal, complication that has been associated with inflammatory myopathies (Table 1). Orrell et al. in a paper in 1998 discussed two cases in Rochester, NY, where patients with DM developed abdominal hematomas [7]. In the first case, a patient with biopsy proven DM for 11 years was started on IVIG monthly for active DM despite steroids and azathioprine. Five years later while on this regimen she developed acute left upper abdominal pain with Hgb drop from 12.9 to $9.1 \mathrm{~g} / \mathrm{dL}$ and was found to have a large left rectus abdominis muscle hematoma. The patient did well with blood transfusion. The second patient was 11 years old with stable DM diagnosed at the age of 8 who presented with pain in the right hip for 3 days. She was taken to the OR for presumed appendicitis but was found to have retroperitoneal bleeding located from a hematoma posterior to the ascending colon and did well after surgery. A third case report by Fang et al. in Kaohsiung, Taiwan, 2008, described a 65-year-old female patient with polymyositis who developed retroperitoneal bleeding from the superior duodenal-pancreatic artery after a flare of her myopathy [8]. Although the bleeding stabilized with angiography, rebleeding occurred in the superior mesenteric artery that was again stabilized by angiography, but the patient expired from sepsis 10 days later. A fourth case presented by Yamagishi et al. in Niigata, Japan, in 2008 described a 64-year-old female with newly diagnosed DM with ILD who developed bleeding in her right psoas and iliacus muscles after 10 days of treatment with pulse steroids, oral cyclosporine, and IV 


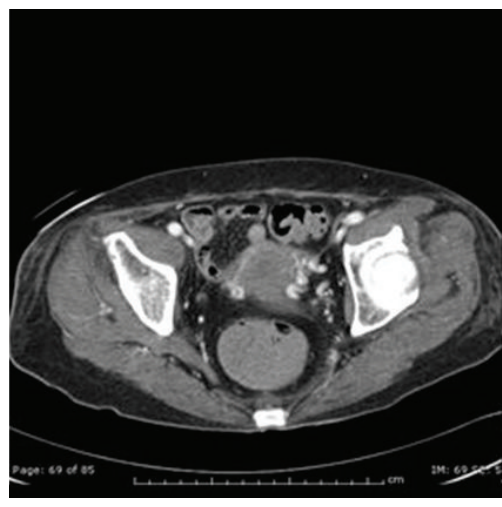

(a)

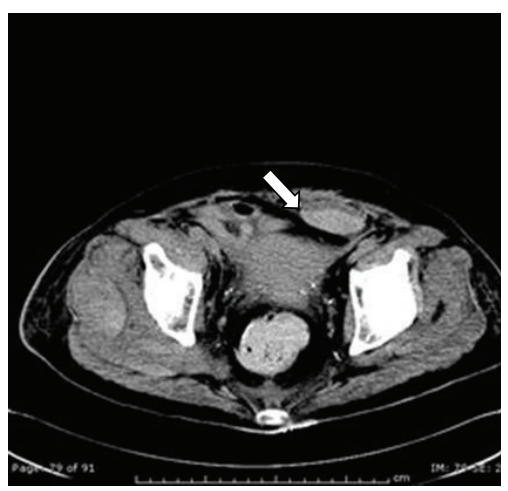

(d)

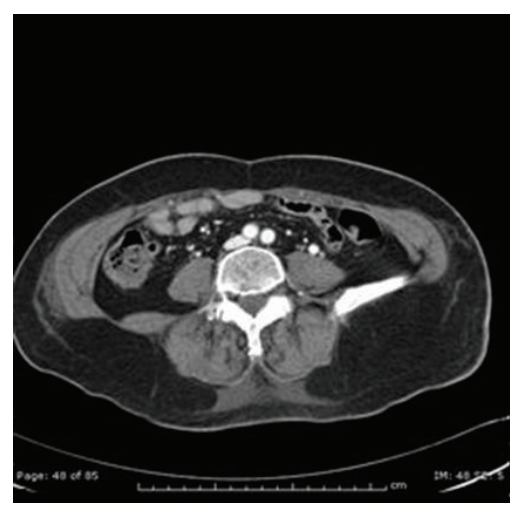

(b)

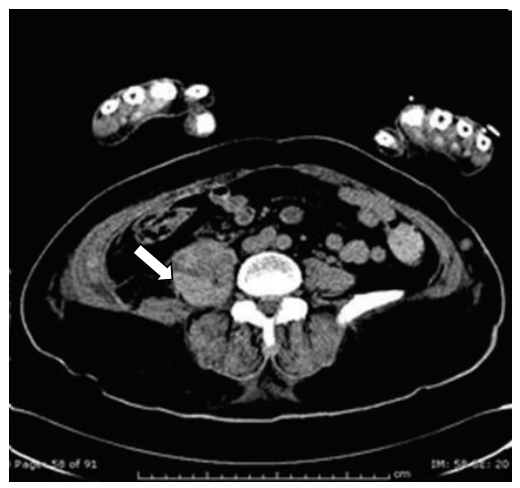

(e)

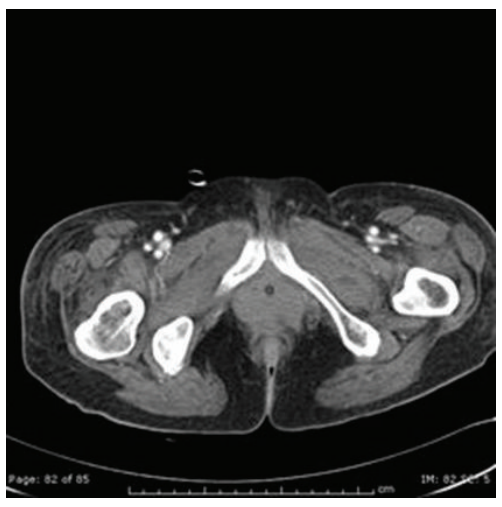

(c)

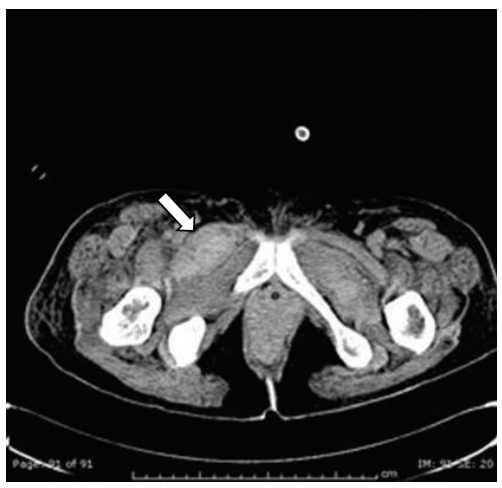

(f)

Figure 8: CT scan of the abdomen and pelvis before and after bleeding. (a-c) CT scan with contrast shows (a) normal rectus, (b) normal iliopsoas, and (c) normal pectineus muscles. (d-f) CT scan without contrast shows bleeding into (d) rectus sheath (arrow), (e) iliopsoas (arrow), and (f) pectineus muscles (arrow).

cyclophosphamide [9]. Angiography localized the lesions to small aneurysms in the retroperitoneum that were embolized, with rebleeding occurring a week later that was again stopped by angiography, but the patient expired weeks later from TTP and multiorgan failure. Other case reports exist in the literature for hemorrhagic myositis, with a report from Higashi et al. in 2009 on a 77-year-old patient with DM who was on heparin for unstable angina [10].

Both of the cases presented in our study were consistent with dermatomyositis (and probably the antisynthetase syndrome) based on clinical features of rash, mechanic's hands, muscle weakness, ILD, elevated muscle enzymes, abnormal EMG/MRI (Case 2 only), and positive antibodies to anti-RO/SSA-52 from the same reference lab. The sites of bleeding in Case 1 (iliopsoas) and in Case 2 (rectus sheath and iliopsoas) were areas frequently reported in the other case reports. The finding of bleeding frequently in these areas but not in other muscle groups seems to be a possible unique finding in hemorrhagic myositis with inflammatory myopathy.

Both patients were given IVIG infusion for rapidly progressive disease. In Case 1, IVIG preceded the bleeding by several days and in Case 2 it followed directly the next day after IVIG infusion. In the past case reports, only one patient was receiving IVIG and had been doing so monthly for 5 years before the bleeding event. Reports of IVIG causing massive intravascular hemolysis are known but are rare, with the usual hemolysis reported as mild and self-limited [11]. Review of the literature did not show an association with IVIG bleeding into the muscle. More frequently reported IVIG complications include renal failure, anaphylaxis, aseptic meningitis, and thrombosis (up to $13 \%$ in one series of patients with autoimmune disorders) [12]. In that same review, it was notable that IVIG thrombotic events favored more arterial involvement (MI, CVA, and peripheral arteries) perhaps hinting at a possible role for enhanced arterial clotting or injury that could relate to hemorrhagic events in our cases.

Finally, both of our cases tested positive for anti-Ro/SSA52 antibody, which is included among the myositis associated antibodies (MAA) [13]. Among autoantibodies in autoimmune disease, the Ro/SSA (made up of Ro60 and Ro52) antibody is the most common one to extractable nuclear antigens. Antibodies to Ro-52 (a tripartite motif protein/TRIM) can be found independent of antibodies to Ro-60 (a small cytoplas$\mathrm{mic}$ ribonucleoprotein/scRNP) in certain autoimmune disorders. Expression of isolated anti-Ro52 alone is mainly seen in certain connective tissue diseases (including myositis), but with unclear clinical significance with an overall prevalence of $0.5 \%$ [14]. The anti-Ro52 antibody occurs in about $30 \%$ 
TABLE 1: Clinical features of selected case reports of hemorrhagic myositis in inflammatory myopathies.

\begin{tabular}{|c|c|c|c|c|c|c|c|}
\hline Author & $\begin{array}{l}\text { Age (yrs) } \\
\text { (at event) }\end{array}$ & Gender & $\begin{array}{c}\text { Clinical history } \\
\text { and prebleeding } \\
\text { therapies }\end{array}$ & $\begin{array}{l}\text { Antibody } \\
\text { profile }\end{array}$ & Site of bleeding(s) & Treatment & $\begin{array}{c}\text { Outcome } \\
\text { (as reported) }\end{array}$ \\
\hline Orrell et al. [7] & 50 & Female & $\begin{array}{c}\text { DM age } 34 \\
\text { Corticosteroid } \\
\text { Azathioprine } \\
\text { IVIG }\end{array}$ & $\begin{array}{c}\text { None } \\
\text { specified }\end{array}$ & $\begin{array}{l}\text { Left rectus } \\
\text { abdominis }\end{array}$ & $\begin{array}{c}\text { Blood } \\
\text { transfusions } \\
\text { Prednisone } \\
\text { Azathioprine }\end{array}$ & $\begin{array}{l}\text { Survived, no } \\
\text { recurrent bleeding }\end{array}$ \\
\hline Orrell et al. [7] & 11 & Female & $\begin{array}{l}\text { DM age } 8 \\
\text { Prednisone }\end{array}$ & $\begin{array}{l}\text { ANA 1:320 } \\
\text { speckled }\end{array}$ & $\begin{array}{l}\text { Retroperitoneum, } \\
\text { posterior to right } \\
\text { ascending colon }\end{array}$ & Supportive & $\begin{array}{l}\text { Survived without } \\
\text { recurrence }\end{array}$ \\
\hline Fang et al. [8] & 65 & Female & $\begin{array}{c}\text { PM age } 65 \\
\text { Hydrocortisone } \\
\text { Prednisone }\end{array}$ & $\begin{array}{l}\text { ANA 1:320 } \\
\text { speckled }\end{array}$ & $\begin{array}{c}\text { Retroperitoneum, } \\
\text { superior duodenal } \\
\text { pancreatic artery, } \\
\text { SMA }\end{array}$ & $\begin{array}{l}\text { Angiography with } \\
\text { embolization }\end{array}$ & $\begin{array}{l}\text { Expired from } \\
\text { sepsis without } \\
\text { rebleeding }\end{array}$ \\
\hline Yamagishi et al. [9] & 64 & Female & $\begin{array}{c}\text { DM/ILD age } 64 \\
\text { Solumedrol } \\
\text { Prednisone } \\
\text { Cyclosporine } \\
\text { Dalteparin }\end{array}$ & $\begin{array}{l}\text { ANA positive } \\
\text { Elevated } \\
\text { serum KL-6 }\end{array}$ & $\begin{array}{l}\text { Retroperitoneum, } \\
\text { right psoas, } \\
\text { right iliacus, } \\
\text { left rectus sheath }\end{array}$ & $\begin{array}{c}\text { Angiography with } \\
\text { embolization with } \\
\text { rebleeding and } \\
\text { repeat } \\
\text { embolization }\end{array}$ & $\begin{array}{c}\text { Expired from TTP } \\
\text { and multiorgan } \\
\text { failure }\end{array}$ \\
\hline Higashi et al. [10] & 77 & Female & $\begin{array}{l}\text { DM age } 77 \\
\text { Solumedrol } \\
\text { Prednisone } \\
\text { Cyclosporine } \\
\text { Heparin }\end{array}$ & $\begin{array}{c}\text { Elevated } \\
\text { serum KL-6 }\end{array}$ & $\begin{array}{l}\text { Retroperitoneum, } \\
\text { left iliopsoas, } \\
\text { left iliacus, } \\
\text { right sternomastoid }\end{array}$ & Supportive & Expired from DIC \\
\hline Van Gelder et al. & 50 & Male & $\begin{array}{c}\text { DM/ILD age } 50 \\
\text { Solumedrol } \\
\text { IVIG }\end{array}$ & Anti-Ro52 & $\begin{array}{l}\text { Retroperitoneum, } \\
\text { right iliacus, } \\
\text { right psoas }\end{array}$ & $\begin{array}{c}\text { Transfusions } \\
\text { Solumedrol }\end{array}$ & $\begin{array}{l}\text { Expired from } \\
\text { multiorgan failure }\end{array}$ \\
\hline Van Gelder et al. & 63 & Female & $\begin{array}{c}\text { DM/ILD age } 63 \\
\text { Solumedrol } \\
\text { IVIG }\end{array}$ & $\begin{array}{l}\text { Anti-SSA } \\
\text { Anti-Ro52 }\end{array}$ & $\begin{array}{l}\text { Retroperitoneum, } \\
\text { right pectineus, } \\
\text { right iliopsoas }\end{array}$ & $\begin{array}{l}\text { Transfusions } \\
\text { Solumedrol } \\
\text { Cytoxan }\end{array}$ & $\begin{array}{l}\text { Expired from } \\
\text { multiorgan failure }\end{array}$ \\
\hline
\end{tabular}

of patients with inflammatory myopathy and up to $72 \%$ of those that are also positive for anti-Jo-1, a myositis specific antibody (MSA) associated with ILD. However, since both our cases were negative for selected MSAs including antiJo-1, it is unclear whether the positive testing for anti-Ro52 may be associated with inflammatory myopathy and ILD or possibly overlapping with additional, nondetected antibodies more specific for inflammatory myopathy associated with this serious complication.

\section{Conclusion}

In summary, we present two cases of inflammatory myopathy (dermatomyositis with ILD) complicated by severe hemorrhagic myositis. The pattern of bleeding in our cases, as in the previous literature, seems to favor the retroperitoneum or rectus abdominis muscles. The possibility of hemorrhagic myositis should always be entertained as a possible complication of inflammatory myopathy, especially in the setting of an acute drop in the hemoglobin. It remains to be seen if the anti-Ro52 antibody may play an accessory role in predicting which patients may be at risk for this potentially very serious complication.

\section{Conflict of Interests}

The authors declare that there is no conflict of interests regarding the publication of this paper.

\section{References}

[1] A. Bohan and J. B. Peter, "Polymyositis and dermatomyositis," The New England Journal of Medicine, vol. 292, no. 7, pp. 344347, 1975.

[2] L. A. Love, R. L. Leff, D. D. Fraser et al., "A new approach to the classification of idiopathic inflammatory myopathy: myositis-specific autoantibodies define useful homogeneous patient groups," Medicine, vol. 70, no. 6, pp. 360-374, 1991.

[3] T. Mimori, Y. Imura, R. Nakashima, and H. Yoshifuji, "Autoantibodies in idiopathic inflammatory myopathy: an update on clinical and pathophysiological significance," Current Opinion in Rheumatology, vol. 19, no. 6, pp. 523-529, 2007.

[4] I. N. Targoff, "Autoantibodies and their significance in myositis," Current Rheumatology Reports, vol. 10, no. 4, pp. 333-340, 2008.

[5] E. Katzap, M. L. Barilla-Labarca, and G. Marder, "Antisynthetase syndrome," Current Rheumatology Reports, vol. 13, no. 3, pp. 175-181, 2011. 
[6] A. J. Rodrigues, M. Jacomelli, P. R. Scordamaglio, and V. R. Figueiredo, "Spontaneous pneumomediastinum associated with laryngeal lesions and tracheal ulcer in dermatomyositis," Revista Brasileira de Reumatologia, vol. 52, no. 5, pp. 796-799, 2012.

[7] R. W. Orrell, H. M. Johnston, C. Gibson, R. M. Cass, and R. C. Griggs, "Spontaneous abdominal hematoma in Dermatomyositis," Muscle \& Nerve, vol. 21, no. 21, pp. 1800-1803, 1998.

[8] T.-J. Fang, S.-C. Hsu, M.-C. Shih et al., "Spontaneous retroperitoneal hemorrhage in a mediastinal tumor in a patient with polymyositis: a case report," Kaohsiung Journal of Medical Sciences, vol. 24, no. 8, pp. 436-440, 2008.

[9] M. Yamagishi, S. Tajima, A. Suetake et al., "Dermatomyositis with hemorrhagic myositis," Rheumatology International, vol. 29, no. 11, pp. 1363-1366, 2009.

[10] Y. Higashi, K. Mera, T. Kanzaki, and T. Kanekura, "Fatal muscle haemorrhage attributable to heparin administration in a patient with dermatomyositis," Clinical and Experimental Dermatology, vol. 34, no. 3, pp. 448-449, 2009.

[11] M. Mohamed, G. Bates, and B. Eastley, "Massive intravascular haemolysis after high dose intravenous immunoglobulin therapy," British Journal of Haematology, vol. 160, no. 5, p. 570, 2013.

[12] I. Marie, G. Maurey, F. Hervé, M.-F. Hellot, and H. Levesque, "Intravenous immunoglobulin-associated arterial and venous thrombosis: report of a series and review of the literature," The British Journal of Dermatology, vol. 155, no. 4, pp. 714-721, 2006.

[13] C. Defendenti, F. Atzeni, M. F. Spina et al., "Clinical and laboratory aspects of Ro/SSA-52 autoantibodies," Autoimmunity Reviews, vol. 10, no. 3, pp. 150-154, 2011.

[14] B. Hervier, M. Rimbert, F. Colonna, M. A. Hamidou, and M. Audrain, "Clinical significance of anti-Ro/SSA-52 kDa antibodies-a retrospective monocentric study," Rheumatology, vol. 48, no. 8, pp. 964-967, 2009. 


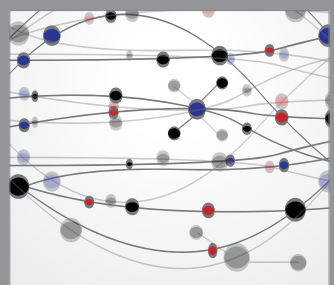

The Scientific World Journal
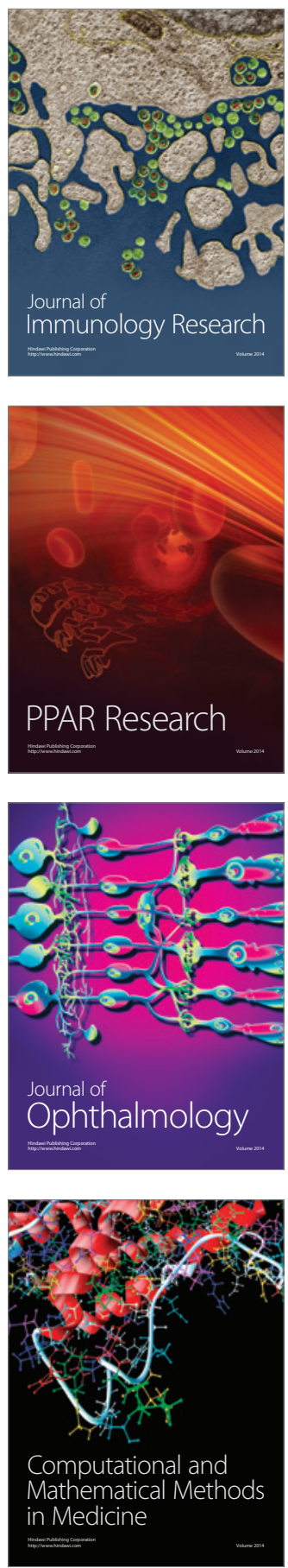

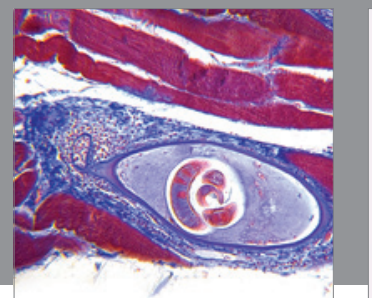

Gastroenterology

Research and Practice
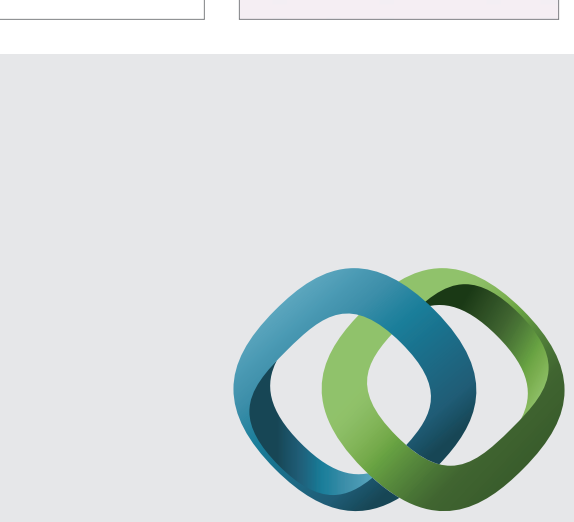

\section{Hindawi}

Submit your manuscripts at

http://www.hindawi.com
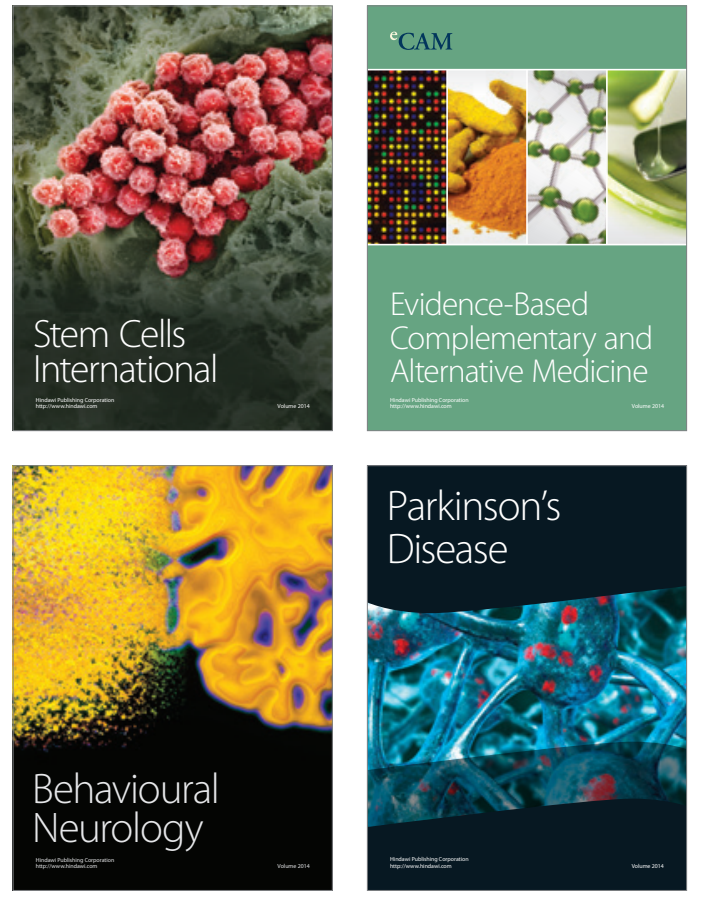
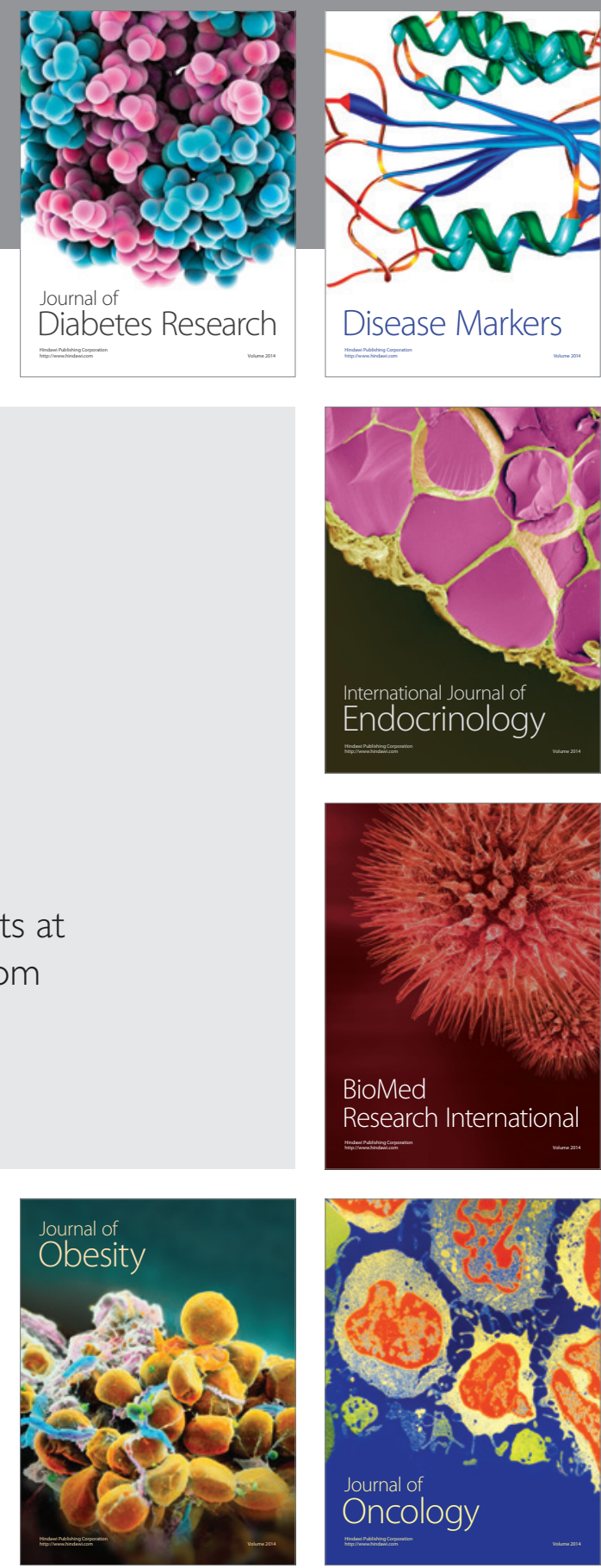

Disease Markers
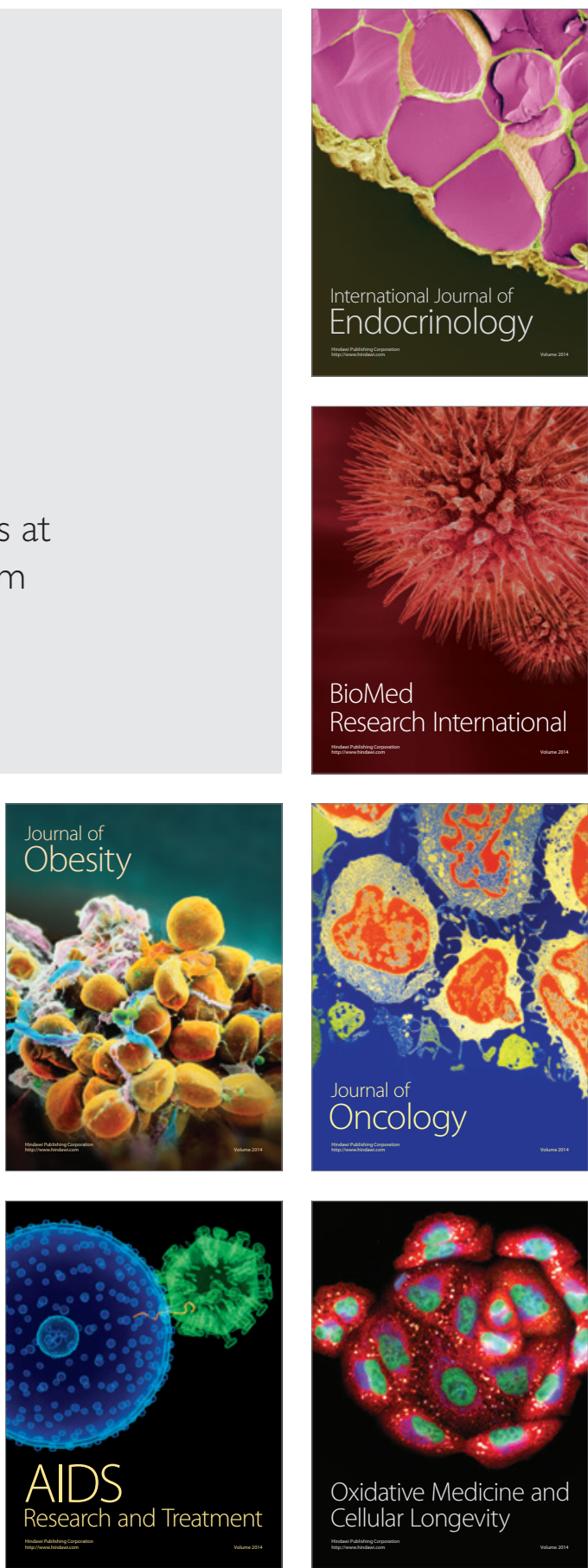